\title{
OBESIDADE: SAÚDE, DOENÇA E EFEITOS DO TREINAMENTO
}

Tadeu João Ribeiro Baptista* Anderson Miguel da Cruz**

\section{RESUMO}

O objetivo desse artigo é discutir a obesidade e suas implicações enquanto saúde e doença, pensando ainda no papel do treinamento como um coadjuvante na redução do peso corporal.

PALAVRAS-CHAVE: Obesidade - Saúde - Doença - Treinamento.

\section{INTRODUÇÃO}

O corpo do ser humano é provavelmente uma de suas principais conquistas. Não por ter capacidades que necessariamente sejam iguais ou maiores do que as de alguns animais, mas, por possuir um cérebro altamente desenvolvido graças ao trabalho realizado desde a pré-história. Poderíamos especular que filogenicamente o ser humano é menos aparelhado que um grande coletivo de animais, para responder a determinadas situações ambientais.

Esse desenvolvimento não se deu de maneira linear. Foram necessárias outras adaptações corporais, entre as quais destaca-se a retenção de tecido específico armazenado sob a pele (adiposo), com pelo menos duas funções fundamentais: a) armazenar energia; b) funcionar como camada de proteção térmica.

Uma terceira função que pode ser atribuída ao tecido gorduroso é o de funcionar como uma espécie de coxim amortecedor ou sistema antichoque para uma grande quantidade de estruturas anatômicas, entre elas tecidos importantes como o cérebro, supra-renal, ovários.

Além das condições fisiológicas, a quantidade de gordura também contribui, em algumas culturas, para o estabelecimento de

\footnotetext{
* Doutorando em Educação pela UFG, Professor da UCG e da ESEFFEGO.

** Professor Doutorando em Bioquímica pela Universidade Federal de Uberlândia, Professor da Universidade Estadual de Goiás , Unidade ESEFFEGO, diretor científico da APCE.
} 
certos padrões sociais, aspectos percebidos, por exemplo, na China e no Japão (WOODMAN, 1995). Kleinubing (1999) citando Franscisch, afirma que a obesidade é provavelmente o mais antigo distúrbio metabólico, observadas ocorrências em múmias egípcias e esculturas gregas.

Voltando aos aspectos biológicos, sobretudo na área da fisiologia do exercício, existem critérios bastante claros do que seria a quantidade de gordura desejada para que uma pessoa possa ter "boa saúde". Segundo o critério de referência proposto por Benke, a quantidade de gordura normal, considerando-se a gordura essencial e a gordura de reserva, aproxima-se de 15\% para o sexo masculino e de $27 \%$ para o sexo feminino, de acordo com McArdle, Katch e Katch (1998) ${ }^{1}$.

Além disso, existem outros índices que são usados como padrão em alusão ao nível de normalidade da quantidade de gordura corporal. Este é o índice de massa corporal, (IMC, $\mathrm{kg} / \mathrm{m}^{2}$, ou Índice de Quetelet) ${ }^{2}$. Parâmetros considerados pela Organização Mundial de Saúde (OMS) podem ser visualizados na figura $\mathrm{I}^{3}$.

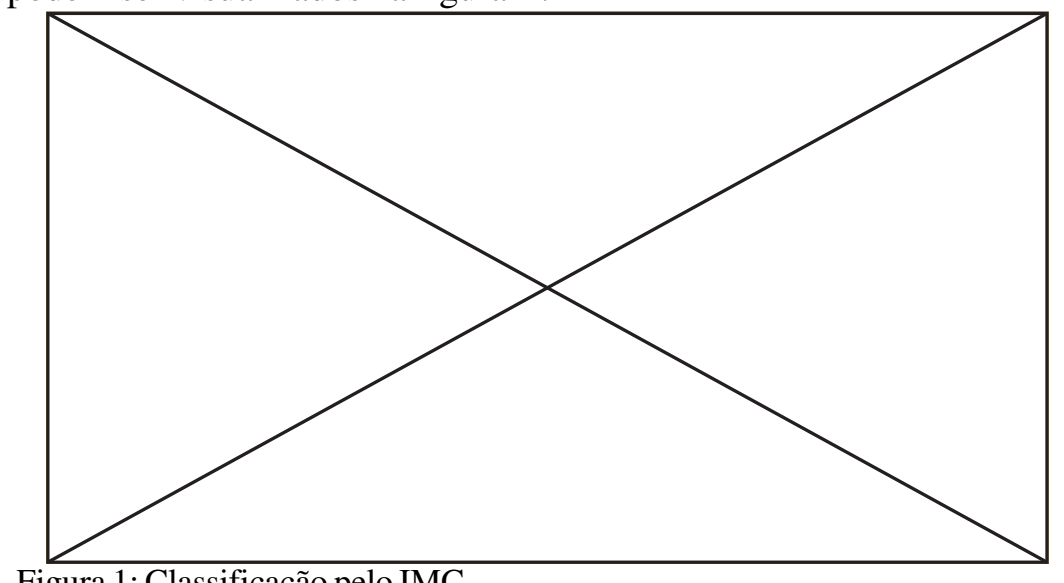

Figura 1: Classificação pelo IMC

Os valores aqui apresentados, assim como alguns outros que poderiam ser discutidos, trazem embutidas pelo menos duas idéias nesse processo. A primeira, a de que existe um ser humano de referência para todos os outros, a outra idéia subliminar, presente nesse discurso, é o fato de haver um padrão de normalidade para todos os homens e mulheres do mundo.

Os preconceitos estabelecidos a partir do padrão corporal em 
relação aos obesos dispensam qualquer explicação de sua situação atual ou pregressa, pois, aparentemente, o seu caso é facilmente entendido por três possibilidades: as disfunções hormonais; o excesso de ingesta de alimentos ou a falta de realização de atividades físicas.

No primeiro caso, a obesidade pode ser considerada uma doença, uma vez que o mau funcionamento de algumas glândulas, como a tireóide, pode reduzir o metabolismo humano, que é a corrente cada vez maior, de pesquisadores que apresentam a teoria da herança genética para a obesidade. Hirsh \& Leibel, citados em Kleinubing (1999), apontam que cinco genes têm sido identificados como possíveis causadores da obesidade. Um destes genes recebe uma denominação especial, é reconhecido como "ob" e codifica a produção de uma enzima denominada de leptina ${ }^{4}$. Esta proteína, após codificação, leva informações para o sistema nervoso central refletindo nos perfis de depósitos de gordura corporal. Para a manutenção do ciclo menstrual regular são necessários níveis mínimos desta proteína, estando esta ainda relacionada com amenorréia de mulheres atletas.

$\mathrm{Na}$ segunda e na terceira situações, normalmente relaciona-se o excesso de gordura e de peso à falta de força de vontade, ao egoísmo e à preguiça. Nessa situação, ele normalmente tem que procurar compensar a sua falta de jeito, cumprindo papéis como ser engraçado ou forte, de acordo com a situação.

Todavia, torna-se intrigante o fato de que, independentemente da "causa da obesidade", esse estado é sempre considerado uma doença, e de acordo com informações de Bankoff (2000), 95\% dos obesos o são por obesidade exógena, ou seja, por balanço calórico positivo, no qual a ingestão de alimentos é maior do que o gasto energético diário. Os $5 \%$ restantes são considerados obesos endógenos, sendo o excesso de peso relacionado a problemas hormonais.

Há ainda uma outra investigação possível com a obesidade do ponto de vista psicológico. Um estudo feito por Woodman (1995), em 20 mulheres obesas, demonstra um componente psíquico extremamente importante no desenvolvimento do excesso de peso.

Ao analisar esses diferentes aspectos, chama a atenção o fato de pelo menos, teoricamente, $95 \%$ dos casos sejam de obesidade exógena. Considerando os fatores psicológicos como endógenos, uma dieta balanceada de acordo com as atividades diárias e a realização de algumas 
atividades físicas poderiam resolver o problema, sendo assim, relativamente fácil resolver esse desequilíbrio. Se o problema é relativamente fácil de resolver, não é possível apenas considerá-lo falta de um padrão corporal que atenda às exigências sociais?

Essa resposta não parece assim tão fácil. Em primeiro lugar devese levantar que a incidência de obesidade no Brasil e no Mundo tem crescido significativamente nos últimos anos, a ponto de ser considerada uma epidemia, comum a países desenvolvidos e subdesenvolvidos. Dados sobre a prevalência de obesidade no maior país em extensão da América do Sul, segundo levantamento realizado em 1989, é de 6\% entre as mulheres e de $13 \%$ entre os homens (WHO, 1997). Ainda de acordo com este mesmo estudo, o "Brasil está mudando rapidamente de um problema de déficit dietético para um de regime alimentar em excesso" (WHO, 1997, p. 23) .

Ao que tudo indica, o Brasil vive um momento identificado como de transição do ponto de vista alimentar. Para Coutinho (S.d., p. 1) “[...] a obesidade desponta como um problema mais freqüente e mais grave que a desnutrição". Pois, esse processo de modificação do padrão alimentar,

[...] sobrecarrega nosso sistema de saúde com uma demanda crescente de atendimento a doenças crônicas relacionadas com a obesidade, como o diabetes tipo 2, a doença coronariana, a hipertensão arterial e diversos tipos de câncer. É provável que 200.000 pessoas morram anualmente em decorrência destas complicações na América Latina. (ibid.)

Esses dados demonstram que o Brasil e a América Latina têm modificado o seu padrão alimentar e não necessariamente apresentando maiores incidências de problemas funcionais. Contudo, as pessoas que apresentam essa perspectiva argumentam que o excesso de peso acarreta um aumento nas despesas governamentais com saúde, sobretudo na população de baixa renda.

Ainda, dentro da reflexão a respeito da epidemia de obesidade que assola toda a América Latina, um dos principais documentos nessa área é o Consenso Latino-Americano de Obesidade (CLAO). De acordo com ele, houve um aumento de $53 \%$ na incidência de obesidade no 
Brasil no período de 1974/75 até 1989. Se este ritmo for mantido "[...] todos os brasileiros serão obesos na primeira metade do terceiro milênio." (CLAO, S.d., p. 5)

Ao ser analisada dessa maneira, a obesidade se apresenta como um sério problema de saúde pública, seja pelos gastos por ela provocados, seja pela possibilidade de desenvolvimento de outras doenças associadas. Em outras palavras, a redução do peso gera redução significativa em outros problemas.

Por exemplo: a diminuição de $10 \mathrm{~kg}$ de cada pessoa de um grupo de obesos significa mais de $20 \%$ de queda da mortalidade geral desse grupo, $30 \%$ da mortalidade por diabetes e mais $40 \%$ da mortalidade de câncer de mama, cólon, próstata e endométrio. Assim, o excesso de peso está diretamente associado à expectativa de vida do indivíduo. Estima-se que a prevenção do excesso de peso/ obesidade reduziria a incidência dessas doenças em, no mínimo, 30\%, com uma economia anual para o país de, no mínimo, 1 bilhão de reais. (BANKOFF, 2000, p. 43)

Afinal, o problema está na obesidade em si ou nas doenças dela decorrentes? O que é a doença, o excesso de peso ou os outros problemas associados? E o que é o excesso de peso? Como ele se estabelece?

Do ponto de vista da reflexão sobre o corpo, uma primeira ponderação possível seria identificar qual a idéia filosófica de corpo com a qual a sociedade está lidando. Ter-se-ia três respostas possíveis. A primeira, refere-se à concepção de corpo como máquina universal e generalizável, voltada para o desempenho e a saúde fisiológica. Outra perspectiva estabelece o contato com o mundo e com ele se relaciona, influenciando e sendo influenciado a partir de sua corporeidade. A terceira e última possibilidade é entender o corpo a partir de suas relações históricas e materiais, que se definem pela classe social da qual este corpo emerge.

Preliminarmente, a resposta sobre o corpo parece se apoiar na primeira concepção, uma vez que a sociedade atual vive regida por idéias como o rendimento e a lucratividade. Tudo isso leva a crer que o problema da obesidade, para os $95 \%$ dos casos exógenos, não se constitui necessariamente como doença e sim como forma de controle sobre as 
pessoas para que elas possam aderir à idéia de magreza (BANKOFF, 2000). Todos esses elementos apontam para o fato de que a obesidade não é em si, somente um problema de saúde pública, mas, uma relação estabelecida a partir de um modelo estético excludente e controlador, ao mesmo tempo em que oferece prazeres associados às facilidades tecnológicas, possibilitando um menor gasto energético, favorecendo o aumento de peso, contrapondo o ideário de um corpo forte, ágil e resistente, tal qual se fazia necessário no século XIX, conforme demonstra Foucault (1999).

\section{OBESIDADE: DEFINIÇÕES E RELAÇÕES COM A SAÚDE}

A obesidade é conhecida desde a pré-história, embora, ao longo dos tempos, ela tenha sido analisada de maneiras diferentes. Na préhistória, ela era símbolo de fertilidade e de representação maternal. $\mathrm{Na}$ medicina greco-romana, era entendida por Hipócrates como causa de infertilidade pelo acúmulo de gordura no útero. Galeno acreditava que ela era consequiência da desobediência à natureza. No período bizantino ela já era conhecida e Cannon de Avicenna, um médico influente da época escreveu o primeiro livro sobre como reduzir a obesidade. A partir do século XVII, as escolas de medicina da época começaram a produzir estudos a respeito da obesidade, a princípio, bastante influenciados pelos estudos de Galeno. No século seguinte, a obesidade era vista como responsabilidade individual e, também, as doenças eram associadas aos miasmas, que alteravam as funções orgânicas, o que se relacionava por sua vez à estagnação do ar. Já no século XIX, os estudos avançam, primeiro, dominados pelos estudos franceses, depois pelos alemães e, finalmente, pelos ingleses. Nesse momento, existem vários estudos relatando os diferentes tipos de obesidade, apoiados sobretudo nos avanços das ciências da época. Finalmente no século XX, os estudos sobre obesidade foram dominados pelos americanos que centraram a terapêutica no controle da ingesta alimentar e no uso de métodos comportamentais (BRAY, 1992).

A obesidade caracteriza-se pelo acúmulo acentuado de tecido adiposo subcutâneo, sendo muitas vezes associada a outras doenças, como a hipertensão, a diabetes, entre outras.

Esse problema pode estar relacionado com o que Mellerowicz \& 
Meller (1987) vão chamar de doenças hipocinéticas. Segundo estes autores,

Devido à falta de atividade física, de trabalho e de treinamento físico, surgiu um novo tipo de doenças deficitárias [...]. Kraus (1961), denominou estas doenças de "hipocinetic diseases" ("hipocinesias"). Elas englobam um complexo amplo de modificações orgânicas e funcionais e sintomas em quase todos os órgãos. Sem dúvida, estes sintomas não se devem somente a um fator, a falta de atividade física, mas a um amplo complexo de fatores patogênicos condicionantes, tais como alimentação excessiva e deficiente, superestimulação nervosa, vícios (fumo) etc. [...] Entre as doenças hipocinéticas encontrase, a obesidade. (MELLEROWICZ \& MELLER, 1987, p. 106)

Dados Estatísticos mostram que o excesso de peso em Goiânia chega a atingir $44,1 \%$ da população, considerados os níveis sobrepeso/obesidade, apesar de haver diferenças entre estes conceitos, os quais já foram discutidos (BAPTISTA, 2000).

Pelo percentual de gordura, o sobrepeso e a obesidade podem ser definidos a partir da figura 2 .

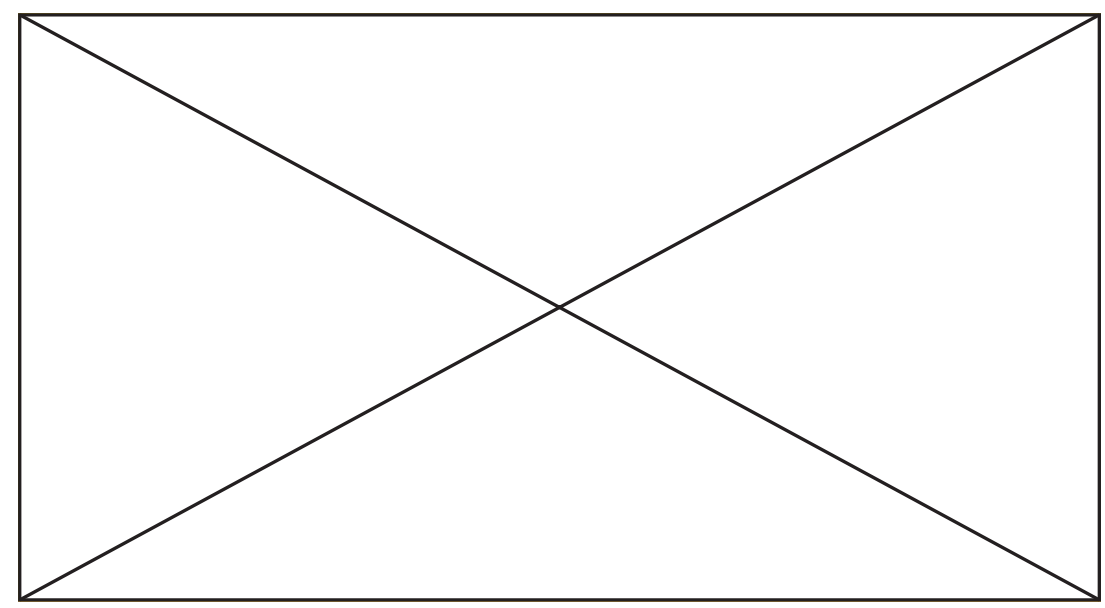

Figura 2: Classificação de Acordo com o \% de gordura. 
Esse fator se justifica devido às diferenças de composição corporal entre homens e mulheres. Aqueles têm maior quantidade de massa muscular, enquanto estas possuem uma maior quantidade de tecido adiposo. Esse aparente excesso no caso das mulheres é responsável pelo controle do ciclo menstrual. Existem evidências de que a redução acentuada do percentual de gordura das mulheres $(<12 \%)$ pode gerar amenorréia, sendo esse um fato relativamente comum na vida de mulheres atletas.

Dowse et al. (1992) demonstra que na população do oceano pacífico a obesidade não é uma situação recente. Nesse mesmo estudo ele demonstra que em um outro estudo,

West notou que a obesidade tem sido considerada de importância etiológica para o diabetes desde tempos mais remotos, e a Comissão de Especialistas da Organização Mundial de Saúde sobre diabetes melito considerou ser a obesidade o fator de risco mais importante para o desenvolvimento de DMNID (DOWSE et al, 1992: 626)

Segundo a WHO (1997), confirmam os dados apresentados por Dowse et al (1992), para a população mundial. Além disso, apresentam outros dados de que, teoricamente, $64 \%$ dos homens e $74 \%$ das mulheres poderiam ter prevenido a Diabetes Melito Não-Insulino Dependente se o seu IMC não tivesse sido maior que $25 \mathrm{~kg} / \mathrm{m}^{2}$.

$\mathrm{O}$ que muitas pessoas consideram o grande problema da obesidade é normalmente ela estar associada a uma maior incidência de problemas cardiovasculares, sobretudo quando existe um alto grau de concentração de gordura na região do abdômen, denominada de gordura andróide ou em maçã. O maior problema, ao que tudo indica, está relacionado ao excesso de células de gordura localizadas sobre o fígado, o que pode comprometer a circulação da via porta (WHO, 1997, p. 48).

A obesidade pode ainda estar relacionada ao aumento da hipertensão, podendo acarretar aumento da prevalência desta doença em 2,9 vezes mais se comparada com pessoas que não apresentam peso excessivo. Existem, também, associações importantes entre um alto percentual de gordura e o desenvolvimento de certos tipos de câncer 
como os de endométrio, ovário e próstata, sobretudo para as pessoas que possuem maior predominância na região do abdômen, em níveis mais baixos de obesidade (idem, p. 52).

Outro problema que pode ser apresentado pelas pessoas obesas diz respeito a problemas na vesícula biliar. Nas pessoas obesas, o risco de pedras na vesícula é três a quatro vezes maior para todas as idades de homens e mulheres quando comparados com pessoas de peso normal, sobretudo, quando há excesso de gordura localizado ao redor do abdômen (idem, p. 53).

O processo de envelhecimento detona no organismo uma modificação estrutural que envolve a diminuição de uma grande massa de tecidos, dentre eles o muscular (sarcopenia), que culmina com a redução da capacidade de produção de força. Simultaneamente acontece um incremento do tecido gorduroso. Distúrbios plurimetatólicos apresentam uma correlação muito estreita com o nível tecidual de gordura, estabelecendo assim uma conexão de morbidade entre gordura, envelhecimento e distúrbios metabólicos.

Não se pode esquecer, no entanto, que um dos fatores de risco para a própria obesidade é o mau funcionamento orgânico, por exemplo, aquele causado por problemas hormonais e que segundo Bankoff (2000) é chamado de obesidade endógena. Raso (2002) em seu artigo referendado, discute a relação de eventos relativos ao envelhecimento e as modificações na capacidade funcional, principalmente de mulheres, relatando que a idade, IMC e peso corporal são fatores que exercem influência na independência social de mulheres acima de 47 anos.

A obesidade é vista atualmente como uma doença associada a outras doenças consideradas graves (SEIDELL et al., 1986). Normalmente, os obesos apresentam altos índices de colesterol sangüíneo, hipertensão e grande possibilidade de desenvolver doenças isquêmicas.

De acordo com Santos (S.d.), seguindo os padrões da Organização Mundial de Saúde (OMS), saúde é o estado de completo bem-estar físico, mental e não apenas ausência de doenças. Acrescenta que é um bem coletivo compartilhado por uma coletividade, determinando assim um locus individual e um coletivo. Este mesmo autor, citando Minayo, ressalta a dependência dos fatores de alimentação, habitação, educação, renda, meio ambiente, trabalho, transporte, lazer, liberdade, acesso à 
posse da terra e aos sistemas de saúde pública. Ainda de acordo com esse autor, o artigo 196 da Constituição Brasileira determina a "saúde" como um direito do cidadão e um dever do Estado. Paiva (1999) defende uma caracterização menos "capitalista" para o conceito de saúde, criando um contínuo que vai de um extremo denominado de "morbidade" até o outro, denominado de "apreciação da vida". Um conceito muito coloquial, mas que confere uma adequação aos moldes sociais atuais.

A necessidade do desempenho motor para a sobrevivência do homem (em seu aspecto mais amplo) fez com que a OMS no final da década de 1980 acrescentasse a seu conceito de saúde o termo aptidão física (GLANER e colaboradores, 1998), usando a definição de Bouchard e colaboradores segundo os quais o desempenho motor tem a finalidade de assegurar a sobrevivência em um determinado ambiente.

Para Monteiro e colaboradores (1999), citando a Organização Pan-americana de Saúde (OPS),

A exemplo do que ocorreu em países desenvolvidos, defende que a redução da miséria em nosso meio é fator fundamental na solução do problema, que passa, por exemplo, pela necessidade de melhoria das condições de moradia, de nutrição e da educação básica destes povos.

Cita também Chadwick: "Homens e mulheres ficam doentes porque são pobres, ficam mais pobres porque são doentes e mais doentes porque são pobres", conclusões que constam de um relatório sanitarista inglês do ano de 1842 (MONTEIRO, 1999).

Para o Programa Brasileiro de Educação em Saúde da Secretaria de Saúde, o conceito atrelado ao desenvolvimento humano é: "O recurso que cada pessoa dispõe para viver, produzir, participar, conhecer e reger sua existência" ${ }^{9}$.

Embora somente nos últimos anos tenha se observado uma mudança singular nos conceitos, preceitos e ações ligadas ao binômio saúde - atividade física (BANKOFF; ZAMAI, 1999), a conexão entre ambos já vem de longos anos, com personagens ilustres da história da 
humanidade, dentre eles o filósofo grego Sócrates (470/469 a.C.), segundo Toscano (1999), foi um indivíduo que veio a óbito com idade próxima a 70 anos, vitimado por envenenamento.

A maioria das doenças tem fatores intermediários em comum que determinam o aparecimento das mesmas, e seguramente trata-se do fator econômico, principalmente ao se fazer uma separação da população por faixas de renda. Para Nunomura e colaboradores (1999), os profissionais de saúde afirmam com certa freqüência uma conexão equilibrada entre os elementos: alimentação, vida familiar e social e prática regular de exercício como fatores fundamentais para uma vida saudável. Afirmam ainda que a qualidade de vida é uma dependência dos subsistemas sociais onde o indivíduo esteja inserido (trabalho, escola, família, clube, ...) sendo uma compensação matemática dos elementos positivos e negativos encontrados em cada um destes ambientes.

"Os anos 90 serão lembrados como a década em que a medicina reconheceu formalmente o fato de a atividade física ser vital para a saúde do corpo." (WILMORE; COSTILL, 2001). Embora somente nos últimos anos é que se tenha sistematizado informações científicas a respeito da influência da atividade física no nível de saúde e qualidade de vida, essa ligação já precede aos registros formais, contudo trata-se de intenções e concepções acientíficas, racistas ou sexistas.

A atividade física, diferente da maioria dos fármacos halopáticos, possui uma ação em todo o organismo, não apenas em alguns sistemas em especial, isto a coloca próximo de uma caracterização holística. Segundo Silva e colaboradores (S.d.), a atividade física está classificada como uma atividade terapêutica não farmacológica.

Do ponto de vista da performance motora, o treinamento desportivo e a medicina desportiva devem exibir uma conexão precisa, uma vez que o máximo rendimento orgânico depende de uma integridade de seus sistemas.

A Educação Física, tendo como campo de estudo o movimento humano, independente de seu paradigma, é sem dúvida uma das "ciências" que deve exibir uma maior conexão com o emprego da atividade física para benefício da saúde.

A despeito da implicância da atividade física e saúde e das ligações profissionais e acadêmicas, não existe até o momento nos cursos regulares de graduação, uma cadeira que responda efetivamente por 
este ramo do conhecimento (BANKOFF; ZAMAI,1999).

O professor de Educação Física não é um profissional reconhecido pelo SUS para o trabalho intra-hospitalar (SANTOS, S.d.). Este autor sugere entre outras as seguintes sub-áreas de atuação do profissional de educação física relativos à saúde:

Administração de programas de reabilitação, manutenção e terapias de saúde

Avaliação (análise) de desenvolvimento físico/mental/desportivo

Atividade física para grupos especiais

Gestantes

Cardiopatas

Idosos

Obesos

Avaliação e orientação vocacional de pacientes com seqüelas orgânicas

Dança hospitalar

Engenharia biomecânica

Farmacologia da atividade física

Ginástica Laboral

O principal motivo que agrega e mantém pessoas em trabalhos físicos é o prazer pessoal (SAMULSKI; NOCE, 2000), mais uma vez refletindo a ligação afetiva com a tarefa a ser cumprida, bem como um estilo de vida. Os mesmos autores encontraram em segunda instância a "melhora da saúde" como fator motivador para a prática desportiva, seguido de "redução de estresse" e "melhora do condicionamento físico".

\section{ALTERAÇÕES MORFOLÓGICAS COM O TREINAMENTO}

Não se tem uma definição final para a etiologia da obesidade. Uma grande quantidade de pesquisas são produzidas no sentido de estabelecer uma conexão genética e fisiológica ao evento e outra parte a uma casualística social, em ambos os casos tendo se produzido resultados positivos. Não se pode deixar de lado o aspecto cultural da morfologia corporal, fenômenos atrelados à corpolatria, nos dias atuais bastante ligados à moda plástica das roupas e às figuras de modelos.

Para diminuição das proporções corporais, o consumo do tecido 
adiposo armazenado é um dos fatores mais importantes, estimular a utilização de gordura como fonte energética passa a ser então a intenção principal dos métodos e sistemas de treinamento. O consumo energético pessoal em diferentes estados metabólicos exerce uma importante ação sobre esta mobilização em atividade e em repouso.

Examinar o gasto energético logo após o trabalho físico realizado não parece muito coerente, uma vez que o metabolismo continua elevado mesmo depois de finalizada a tarefa, sendo denominado atualmente de consumo excessivo de oxigênio. Este retorno pode levar um tempo variável para ser adquirido, dependendo da intensidade da tarefa feita, podendo levar minutos, ou mesmo horas. Possivelmente é em função desta alteração metabólica da recuperação que alguns pesquisadores insistem em afirmar que a musculação consome tecido adiposo ${ }^{10} \cdot{ }^{4}$ Convém lembrar também da acomodação do organismo ao esforço físico, a chamada economia de trabalho, que não é levada em conta algumas vezes, na evolução do treinamento dentro do macrociclo programado. Desta forma, admite-se o maior gasto calórico no período após exercício e/ou aumento da economia de trabalho com a evolução do treinamento, concluindo-se que apenas no início esta variação metabólica poderá influenciar positivamente no processo de consumo de energia.

O metabolismo de repouso representa em torno de 60 a 75\% das calorias totais gastas diariamente. O papel do treinamento físico na Taxa Metabólica de Repouso (TMR), ainda não foi bem esclarecido. Estudos transversais demonstram que se encontram elevadas em corredores de longa distância, quando comparadas a pares morfologicamente semelhantes. Está associado ao tecido isento de gordura, um tecido que citologicamente é mais ativo. Com a evolução do treinamento, um dos principais eventos é a perda de tecido adiposo, e a depender do sistema de treinamento, um aumento da massa muscular, o que resulta por conseqüência em um aumento do percentual de tecido metabolicamente ativo. Este fator contribui para a aceitação do trabalho de força na redução do peso corporal.

Durante o exercício, os ácidos graxos livres são liberados de seus sítios para serem consumidos na produção de energia, tendose sugerido que esta mobilização seja fomentada pelo GH (hormônio do crescimento), corroborada pelas observações de aumentos agudos na concentração do mesmo durante a realização de atividades físicas 
e durante a fase de recuperação da mesma. Pesquisas sustentam que o trabalho aeróbio de baixa intensidade deve ser priorizado em função desta mobilização.

Diante de todo o exposto, a atividade física deve ser repensada quanto à saúde, e sobretudo em relação à obesidade como forma de melhor utilizá-la para se alcançar os objetivos desejados quanto às transformações, sejam elas biológicas ou sociais.

Obesity: health, illness, and the effects of training

\begin{abstract}
The aim of this article is to discuss obesity and its implications on health and illness, and to reflect upon the role of training as a component in body weight loss. KEY-WORDS: Obesity - health - illness - training
\end{abstract}

Obesidad: salud, enfermedad y efectos del entrenamiento.

\title{
RESUMEN
}

El objetivo de este artículo es discutir la obesidad y sus implicaciones relacoinadas a salud y enfermedad, pensando todavía en el papel del entrenamiento con relación a un coadyuvante en la reducción del peso corporal.

PALABRAS-CLAVE: Obesidad - Salud - Enfermedad - Entrenamiento.

\section{NOTAS}

${ }^{1}$ Convém lembrar que a constituição anatômica do ser humano difere grandemente de uma população para outra. A estrutura tecidual de um europeu típico não pode ser simetricamente comparada às distribuições teciduais de um aborígine australiano. Desta forma, as extrapolações estatísticas que tendem a disseminar um perfil numérico efetivo como padrão universal, deve ser interpretado e discutido relevando se tais diferenças constitutivas.

${ }^{2} \mathrm{O}$ chamado Índice de Massa Corporal (IMC) é um parâmetro que progressivamente cai em desuso, principalmente nos locais de práticas desportivas voltadas para alto rendimento, onde as dimensões corporais e volume de massa muscular dos atletas inviabilizam a interpretação 
baseada nas escalas apresentadas para uma população convencional. ${ }^{3}$ Dados adaptados do relatório sobre obesidade de 1997, da Organização Mundial de Saúde (OMS), doravante chamada WHO (World Health Organization).

${ }^{4}$ É uma molécula constituída de 167 aminoácidos, o gene humano que codifica a leptina encontra se no cromossomo 7 , região $7 \mathrm{q} 31.3$. A expressão deste gen é $75 \%$ mais alta em mulheres obesas do que em homens, sugerindo uma influencia dos hormônios sexuais.

${ }^{5}$ Brazil is rapidly shifting from a problem of dietary deficit to one of dietary excess. (WHO, 1997:23)

"“(...) todos los brasileños seran obesos en la primera mitad del tercer milenio. (CLAO, S.d.: 5)

${ }^{7}$ Diabetes Melito Não-Insulino Dependente (DMNID).

${ }^{8}$ West noted that obesity had been considered to be of etiological importance for diabetes since the earliest times, and the World Health Organization Expert Committee on Diabetes Mellitus considered to be the most powerful known risk factor for the development of NIDDM. (Dowse et al, 1992: 626)

${ }^{9}$ Citação obtida no site do ministério da Saúde, creditada a Sylvayn Levi, diretor do Programa de Educação em Saúde na época, ligada á Secretaria de Políticas da Saúde.

${ }^{10} \mathrm{Willmore} \mathrm{e} \mathrm{Costill} \mathrm{apresentam} \mathrm{pensamento} \mathrm{similar} \mathrm{na} \mathrm{página} 678$.

\section{REFERÊNCIAS}

KLEINUBING, Marcos C. Ações dos níveis plasmáticos de leptina e sua influência no controle do peso corporal. Revista eletrônica. Disponível em: <www.efdeportes.com/efd64/leptina.htm>

BANKOFF, Antonia Dalla Pria; ZAMAI, Carlos Aparecido. Estudo do perfil dos professores do ensino fundamental do estado de São Paulo sobre o conhecimento do tema atividade física e saúde. In: Revista Brasileira de Atividade Física e Saúde. v. 4, n 3, 1999.

BANKOFF, Maria A. D. P. Obesidade, adolescência e atividade física e saúde. In: MOREIRA, Wagner W. \& SIMÕES, Regina (Org.). Fenômeno Esportivo no início de um novo milênio. Piracicaba: 
UNIMEP, 2000, pp. 43-52.

BAPTISTA, Tadeu J. R. A Atividade física no projeto C.A.R.M.E.N.T.: um projeto em construção. In: ANAIS DO CONGRESSO ESTADUAL DE EDUCAÇÃO FÍSICA E ESPORTES DE GOIÁS: Sessões Científicas. Goiânia: FIEP, 01 a 04/06/2000, p. 7.

BRAY, George A. Obesity: historical development of scientific and cultural ideas. In: BJORNTORP, Per \& BRODOFF, Bernard N. Obesity. Philadelphia: J.B. Lippincott Company, 1992, pp. 281-293.

CLAO. CONSENSO LATINO-AMERICANO SOBRE OBESIDADE. [S.l.: S.n., S.d.]

DOWSE, Gary et al. Obesity in Pacific Populations. In: BJORNTORP, Per \& BRODOFF, Bernard N. Obesity. Philadelphia: J.B. Lippincott Company, 1992, pp. 619-39.

FOUCAULT, Michel. Microfísica do poder. 14. ed. Rio de Janeiro: Graal, 1999.

GLANER, Maria Fátima e colaboradores. Diagnóstico da aptidão física relacionada à saúde de universitários. In: Revista Brasileira de Atividade Física e Saúde. v.3, n. 4, 1998.

McARDLE, William D.; KATCH, Frank I.; KATCH, Victor L. Fisiologia do exercício: energia, nutrição e desempenho humano. 4. ed. Rio de Janeiro: Guanabara Koogan, 1998.

MELLEROWICZ, H.; MELLER, W. Treinamento físico: bases e princípios fisiológicos. São Paulo: EPU, 1987.

MONTEIRO, Luiz Henrique e Colaboradores. Atividade física e hanseníase: investigação de lesões sensitivo motoras a partir de estudo transversal híbrido no instituto Lauro Souza Lima, Bauru - SP. In: Revista Brasileira de Ciências do Esporte 21(1), setembro de 1999.

NUNOMURA, Myrian e Colaboradores. Nível de estresse, qualidade d

118 BAPTISTA, T.J.R. CRUZ, A.M. Obsidade: daúde, doençca e efeitos ... 
vida e atividade física: uma comparação entre praticantes regulares e ingressantes sedentários. In: Revista Brasileira de Atividade Física e Saúde, v. 4, n. 3, 1999.

PAIVA, Andréa C. de. A educação física promovendo saúde na escola: uma regressão histórica. In: Revista Brasileira de Ciências do Esporte -21(1), setembro de 1999.

RASO, Wagner. A adiposidade corporal e a idade prejudicam a capacidade funcional para realizar as atividades da vida diária de mulheres acima de 47 anos. In: Revista Brasileira de Medicina Desportiva, v. 8, n. 6, nov.- dez. 2002.

SAMULSKI, Dietmar Martin; NOCE, Franco. A importância da atividade física para a saúde e qualidade de vida: um estudo entre professores, alunos e funcionários da UFMG. In: Revista Brasileira de Atividade Física e Saúde, v. 5, n. 1, 2000.

SANTOS, Leonardo José Maturana dos. A educação física hospitalar em desenvolvimento: uma breve apresentação das 32 sub-especialidades de atuação profissional no campo da saúde. Disponível em: < $\underline{\text { http:// }}$ efdeportes.com/revistadigital >. Acesso em: 2003.

SEIDELL, Jacob et al. Overweight and chronic illness - a retrospective cohort study, with a follow-up of 6-17 years, in men and women of initially 20-50 years. In: Journal of Chronic Disease. Great Britain: Pergamon Journals, 1986, v. 39, n. 8, pp. 585-593.

SILVA \& LOPES, Fabrício Carvalho Marques e Colaboradores. Efeito fisiológico imediato de uma atividade física na água, em mulheres com hipertensão. Disponível em: <http://www.efdeportes.com>. Acesso em 2003.

TOSCANO, José Jean de Oliveira. Exortação do filósofo Sócrates sobre a influência do exercício físico na saúde. In: Revista Brasileira de Atividade Física e Saúde. V. 4, n. 3, 1999. 
WHO - WORLD HEALTH ORGANIZATION. Obesity: preventing and managing the global epidemic - report of a WHO consultation on obesity. GENEVA: WHO, 1997.

WOODMAN, Marion. A coruja era filha do padeiro: obesidade, anorexia nervosa e o feminino reprimido. São Paulo: Cultrix, 1995.

Recebido: novembro de 2003 Aprovado: dezembro de 2003

Endereço para correspondência:

Tadeu João Ribeiro Baptista R. 801, Q. 927, L. 03

Jardim Moema - Goiânia-Goiás

CEP: $74633-380$ 